\title{
BMJ Open Association between mothers' screening uptake and daughters' HPV vaccination: a quasi-experimental study on the effect of an active invitation campaign
}

\author{
Francesco Venturelli, ${ }^{1,2,3}$ Flavia Baldacchini, ${ }^{4}$ Cinzia Campari, ${ }^{2,5}$ Cinzia Perilli, ${ }^{6}$ \\ Maria Grazia Pascucci, ${ }^{7}$ Alba Carola Finarelli, ${ }^{7}$ Luigi Moscara, ${ }^{8}$ Paolo Giorgi Rossi ${ }^{1,2}$
}

To cite: Venturelli $F$, Baldacchini F, Campari C, et al. Association between mothers' screening uptake and daughters' HPV vaccination: a quasi-experimental study on the effect of an active invitation campaign. BMJ Open 2017;7:e016189. doi:10.1136/ bmjopen-2017-016189

- Prepublication history and additional material for this paper are available online. To view please visit the journal (http:// dx.doi.org/10.1136/bmjopen2017-016189).

Received 30 January 2017 Revised 28 June 2017 Accepted 28 July 2017

CrossMark

For numbered affiliations see end of article.

\section{Correspondence to}

Dr Francesco Venturelli; venturelli.dr.francesco@gmail. com

\section{ABSTRACT}

Objectives In Emilia-Romagna, the Human Papillomavirus (HPV) vaccination campaign started in 2008 offering free vaccines for 1996 and 1997 cohorts. Systematic active invitation was implemented for the 1997 cohort. Our study aimed at measuring the impact of the active invitation campaign on HPV vaccine coverage and on coverage inequalities in 11-year-old girls. Second, we evaluated the effect of the HPV vaccination campaign on participation in cervical cancer screening by mothers of target girls.

Methods We collected information on vaccination status for girls residing in Reggio Emilia in 2008 and mothers' screening history, before and after the 2008 vaccination campaign. Log-binomial regression models were performed to estimate Relative Risk (RR) and 95\% confidence intervals (Cls) of being vaccinated as regarded citizenship, siblings, mothers' education, marital status and screening history, stratified by birth cohort. We also calculated RR of receiving a Pap test after the vaccination campaign as regarded education, daughter's cohort and mothers' decision to have their daughter vaccinated. Interaction between education and cohort in mothers overdue for Pap testing was calculated.

Results Vaccination coverage was $46.3 \%$ for the uninvited cohort (1046/2260) and $77.9 \%$ for the invited cohort (1798/2307). In the uninvited cohort, daughters' vaccination showed association with mothers' education (8 to 11 years of education vs. graduated mothers, RR $1.6195 \% \mathrm{Cl} 1.14-2.28$ ), citizenship (foreigners vs. Italians, RR $0.4595 \% \mathrm{Cl} 0.37-0.56$ ) and screening history (regular vs. non-participant; RR $1.7295 \% \mathrm{Cl} 1.26-2.36$ ). In the invited cohort, only a slight association with screening history persisted (regular vs. nonparticipant; RR 1.20 95\% Cl 1.04-1.40). Highly educated under-screened mothers of the invited cohort showed a higher probability of receiving a Pap test after the vaccination campaign period (RR 1.27 95\% Cl 1.04-1.56) compared with those not invited,

Conclusion Active invitation could increase overall HPV immunisation coverage and reduce socio-demographic inequalities and the association with mothers' screening participation.

\section{INTRODUCTION}

\section{Background}

HPV is a necessary cause of cervical cancer ${ }^{1}$ and an effective and safe vaccine is now
Strengths and limitations of this study

- The peculiar activation of the HPV vaccine campaign in the Emilia-Romagna Region allowed us to compare two strategies to offer free vaccine (one with active invitation and one without it) with a quasi-experimental study design.

- We could assess both association between mothers characteristics and daughters' vaccine uptake and the impact of the vaccination campaign on mothers' participation in screening.

- As 11-year-old girls represent the target of the vaccination campaign, for the time being we cannot directly assess the impact of vaccination on screening participation.

- Only data on Pap tests performed through public screening programmes were available, while no data on opportunistic screening were available.

available. ${ }^{2}$ The HPV vaccine has been included among the recommended vaccination schedule in the vast majority of industrialised countries. In Italy, it has been included among recommended and free vaccines since 2007 for 11-year-old girls. ${ }^{3}$

Cervical cancer is an oncologic condition against which we have developed the most effective screening strategy. Pap test or HPV test can reduce disease incidence by more than $60 \%$, by identification and treatment of pre-cancerous lesions. ${ }^{4}$ Unfortunately, screening strategies need repeated testing during a woman's life and population coverage turns out often scarce in many countries and regions. On the other hand, vaccines used until now only protect women from two oncogenic HPV types, which are responsible for more than $70 \%$ of cancers in Europe. ${ }^{5}$ Thus, vaccinated women will need to be screened throughout their lives, even if probably less intensively ${ }^{6}$

Some authors hypothesised that vaccinated women might feel completely protected 
therefore considering participation in the screening programme as pointless. ${ }^{7}$ Conversely, other authors hypothesised that vaccination campaigns play a sensitisation role on target women as concerns cervical cancer and HPV-related risks, promoting compliance to screening protocol..$^{8-12}$

Many studies tried to disentangle the complex interactions between vaccination and screening campaigns in determining women's behaviour related to vaccine and screening uptake, both as individuals and mothers of girls in the vaccination target age (figure 1 and figure 2). ${ }^{91013-25}$

Moreover, screening coverage is often influenced by inequalities. Women with low socio-economic status or low educational level, immigrant women or stemming from disadvantaged ethnic groups are more often under- or never screened. ${ }^{11} 122627$ In many countries, vaccinations have low coverage, therefore outreaching strategies such as school-based campaigns and active individual invitations are currently being assessed. ${ }^{28}$ The 2015 supplements of European Guidelines for Quality Assurance in cervical cancer screening reported that organised vaccination campaigns, either school-based or public health centre-based, are the most effective ways to reach the highest vaccination coverage. ${ }^{29}$ An Italian survey showed that regions adopting active invitation by Public Health Authority with fixed appointment for vaccination and more than one recall for non-responders reached wider vaccination coverage. ${ }^{30}$ Other studies analysed secondary prevention, leading to similar findings. In a 2012 systematic review, active invitation with letter led to a higher participation level for cervical, breast and colorectal cancer screening programmes compared with non-invitation, ${ }^{31}$ moreover organised screening programmes decreased the gradient of coverage according to socio-economic status. ${ }^{32}$

The effect of active invitation on reducing health inequalities in HPV vaccination coverage has not yet been assessed. Moreover, it is relevant to assess the association between non-vaccination and non-participation in the screening programme, both potentially related to lack of confidence in the National Health Service. ${ }^{33}$ Indeed, vaccination is going to have an impact on cervical cancer incidence, in particular when targeting girls at higher risk of not being protected by screening in their adulthood. ${ }^{34}$

The Emilia-Romagna vaccination campaign started in 2008 and adopted two different strategies for the first two target cohorts. The 1996 cohort, including 12-year-old girls at that time, was offered free vaccine, although without active invitation following an information campaign. The 1997 cohort, including 11-year-old girls at that time, received active invitation for all girls. This different approach gave us an opportunity to evaluate the impact of intervention with a quasi-experimental study design.

\section{Objective}

The primary objective was to measure the impact of the active invitation campaign on both HPV vaccine coverage in pre-adolescent girls and coverage inequalities in Reggio Emilia province.

Our secondary objective was to assess the impact of the HPV vaccination campaign on participation in cervical cancer screening by mothers of target girls.

\section{METHODS}

\section{Setting}

Reggio Emilia is a province in Emilia-Romagna, an administrative region of north-east Italy. It has a total population of about 550,000. Each 11-year-old cohort comprises about 2300 girls.

In Italy, in March 2007, HPV vaccine was included in the recommended free vaccine schedule for all 11-year-old girls. ${ }^{35}$

Emilia-Romagna regional council started a vaccination campaign in March 2008 (Dgr 236/2008). The 1997 cohort was actively invited. In the same period, the Regional Health Service also provided free vaccine for girls born in 1996, although the campaign did not include active invitation. The vaccination campaign included information through opportunistic advice from paediatricians and information leaflets delivered by Public Health Centres for both cohorts and another information document about HPV vaccine attached to the invitation letter only for the 1997 cohort. Both cohorts maintained the right to be vaccinated for free until the age of 18 years. The vaccine used was bivalent vaccine Cervarix (GlaxoSmithKline Biologicals) with a three-dose schedule: 0, 1, 6 months.

Invitation letters for HPV vaccination were delivered by the Local Health Authority (LHA) Paediatric Department and included demographics, appointment date and times, vaccination centre address, information about the vaccine, vaccination cycle and vaccination procedure, and contact details when a change of appointment was necessary. A leaflet with more detailed information on HPV and cervical cancer was attached. In case a girl missed her first appointment, two reminders were sent, 45 days and 90 days from the invitation letter.

Emilia-Romagna has been implementing cervical cancer screening since 1999, in compliance with Italian Guidelines which recommended 25- to 64-year-old women attending cytology-based screening every 3 years. The entire Reggio Emilia eligible population was regularly invited to a fixed appointment, every 3 years. The 2008 participation rate was $68 \%$. Test coverage was approximately $90 \%$, thanks to spontaneous participation in screening and opportunistic private screening. ${ }^{36}$

\section{Study design and population}

This is a cohort study based on routinely collected data.

Girls' cohorts were set up in accordance with the Reggio Emilia Health Registry, and included all girls living in Reggio Emilia province in 2008 and born between 1996 and 1997. Vaccination archives collected data on vaccinations by public clinics. 

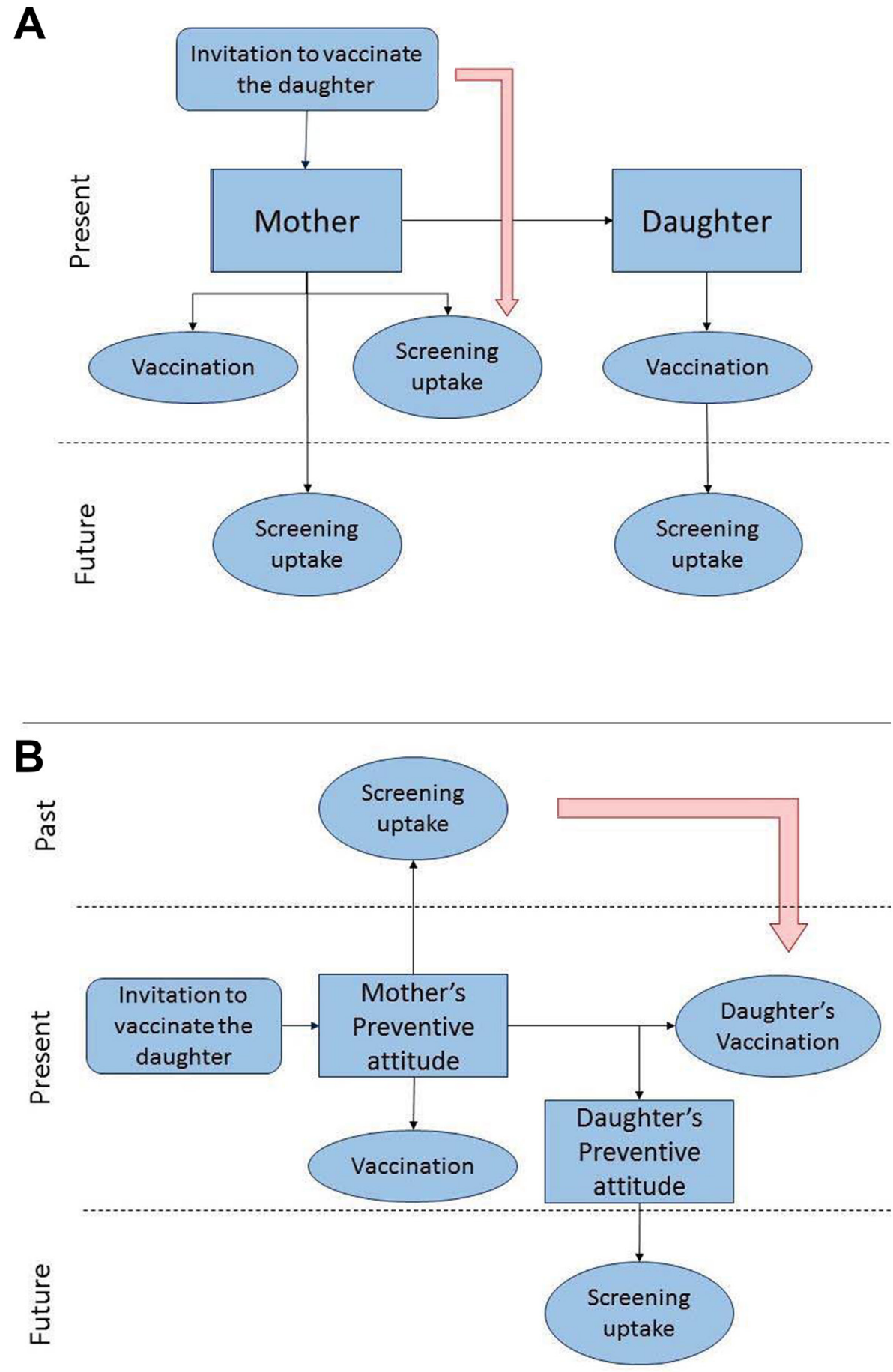

Figure 1. Conceptual model for interpreting the relations between vaccination and screening uptake. (A) Possible effects of vaccination campaign on screening uptake. Vaccination could influence the future participation in screening in adults receiving the vaccine when already eligible for screening but invitation to vaccinate the daughter can act as a reminder for screening (left side); the mother's decision on vaccinating the daughter can also influence the future participation in screening of the daughter. (B) Possible association between vaccination and screening history. In this case the preventive attitude of the mother influenced her probability of having previously participated in screening, of being vaccinated now or in the future and the choice to vaccinate the daughter, but can also influence the preventive attitude of the daughter, thus the propensity of the daughter to participate in screening in the future. The red arrows show the relations assessed in the present study.

By record linkage of the Health Service Population Registry and vaccination archives (key: name, surname, date of birth) we defined vaccination status for each girl, including vaccines administered between 2008 and 2011. In Emilia-Romagna, vaccinations devoted to 12-year-old individuals were run by Public Health clinics, as family paediatricians or GPs were not involved in the vaccine administration. Vaccination registration by the Public Health Service was mandatory, all doses being tracked. 


\begin{tabular}{|c|c|c|c|c|c|c|}
\hline \multicolumn{2}{|r|}{ "Mother on Mother" } & \multicolumn{5}{|c|}{ Effect of HPV vaccination status on cervical cancer screening participation in young adults } \\
\hline \multirow{2}{*}{ ă } & & FIRST AUTHOR; YEAR & COUNTRY & STUDY DESIGN & POPULATION & RESULTS (at least one dose Vs. 0 doses) \\
\hline & & (Palmer, 2016)[13] & Scotland & Cohort & 201,023 & $\mathrm{RR}=1.22(95 \% \mathrm{CI} 1.20-1.26)$ (only 1 Vs. 0 doses) \\
\hline & \multirow{4}{*}{\begin{tabular}{|l|l} 
Mother's \\
Preventive \\
attitude
\end{tabular}} & (Boone, 2016)[14] & USA & Case control & $\begin{array}{c}1123 \text { cases; } \\
1123 \text { controls }\end{array}$ & $\mathrm{HR}=2.98(95 \% \mathrm{CI} 2.45-3.61)$ (only 1 Vs. 0 doses $)$ \\
\hline & & (Sauer, 2015)[10] & USA & Cross-sectional & 7095 & $\mathrm{PR}=1.08(95 \% \mathrm{CI} 1.04-1.11)$ \\
\hline & & (Paynter, 2015)[15] & USA & Cohort & 27786 & $\begin{array}{l}\mathrm{OR}=1.36(95 \% \mathrm{CI} 1.16-1.61) \\
\mathrm{Adj} \mathrm{OR}=0.82(95 \% \mathrm{CI} 0.67-1.02)\end{array}$ \\
\hline \multirow[b]{2}{*}{ 惹 } & & (Herweijer, 2015)[9] & Sweden & Cohort & 629,703 & $\begin{array}{l}\mathrm{HR}=1.28(95 \% \mathrm{CI} 1.24-1.32)_{i} \\
\mathrm{Adj} H R=1.05(95 \% \mathrm{CI} 1.02-1.08)\end{array}$ \\
\hline & & (Budd, 2014)[16] & Australia & Cross-sectional & 2.7 million & $\begin{array}{l}\mathrm{PR}=0.79(95 \% \mathrm{CI}, 0.78-0.80) ; 20-24 \text { years old } \\
\mathrm{PR}=0.77(95 \% \mathrm{CI}, 0.76-0.78) ; 25-29 \text { years old }\end{array}$ \\
\hline & "Mother on Daughter" & \multicolumn{5}{|c|}{ Effect of mothers' cervical cancer screening history on daughter's HPV vaccination (at least one dose) } \\
\hline \multirow{3}{*}{ 范 } & \multirow{3}{*}{$\begin{array}{c}\text { screening } \\
\text { uptake }\end{array}$} & FIRST AUTHOR; YEAR & COUNTRY & STUDY DESIGN & POPULATION & RESULTS (Pap test Vs no Pap in last 3-5 years) \\
\hline & & Present study & Italy & Cohort & 1445 & $\begin{array}{l}\text { Adj } R R=1.20(95 \% \text { CI 1.04-1.40) Vaccine active invitation } \\
\text { Adj } R R=1.72(95 \% \text { CI1.26-2.36) No active invitation }\end{array}$ \\
\hline & & (Hansen, 2015)[17] & Norway & Cohort & 90,842 & Adj $\mathrm{OR}=1.16(95 \% \mathrm{CI} 1.10-1.23)$ \\
\hline & \multirow{2}{*}{$\begin{array}{l}\text { Mother's } \\
\text { Prevertive } \\
\text { attitude }\end{array}$} & (Markovitz, 2014)[18] & USA & Cross-sectional & 38,604 pairs & Adj $\mathrm{OR}=1.07(95 \% \mathrm{CI}=1.06-1.08)$ \\
\hline & & (Steens, 2013)[19] & Netherlands & Cross-sectional & 337,368 & Adj OR=1.54 (95\%CI 1.51-1.57) \\
\hline & Daughter's & (Spencer N P, 2013)[20] & England & Cohort & 117,343 & $\begin{array}{l}\text { Adj OR= } 3.5(95 \% \text { CI } 3.1-4.0) \text { Routine } 12 \text { yo } \\
\text { Adj OR }=2.1(95 \% \text { CI 1.8-2.4) Catch up }\end{array}$ \\
\hline & & (Hechter, 2013)[21] & USA & Cohort & 254,489 Boys & $\mathrm{RR}=1.13(95 \% \mathrm{CI} 1.01-1.26)$ \\
\hline \multirow{3}{*}{ 总 } & & (Lutringer-Magnin, 2013)[22] & France & Cross-sectional & 502 & Adj OR= $6.2(95 \%$ CI $1.5-25.8)$ \\
\hline & & (Lefevere, 2011)[23] & Belgium & Cohort & 127,854 & Adj OR= $=4.5(95 \%$ CI $3.5-5.9)$ \\
\hline & & $($ Chao, 2009)[24] & USA & Cross-sectional & 148,350 & $\mathrm{OR}=1.47(95 \% \mathrm{CI} 1.43-1.52)$ \\
\hline \multicolumn{2}{|r|}{ "Daughter on Mother" } & \multicolumn{5}{|c|}{ Effect of Daughters' active invitation for HPV vaccination on cervical cancer screening participation of under-screened mothers } \\
\hline \multirow{3}{*}{ 莒 } & $\begin{array}{l}\text { Invitation to vaccine } \\
\text { the daughter }\end{array}$ & FIRST AUTHOR; YEAR & COUNTRY & STUDY DESIGN & POPULATION & RESULTS (Invited Daughters Vs no invitation) \\
\hline & \multirow{2}{*}{ Mother } & Present study & Italy & Cohort & 1445 & $\begin{array}{l}\text { Adj } R R=1.04(95 \% \mathrm{CI} 0.96-1.13) \text { overall, overdue for Paptest; } \\
\operatorname{Adj} R R=1.27(95 \% \mathrm{CI} 1.04-1.56) \text { gradhate, overdue for Paptest }\end{array}$ \\
\hline & & (Spencer, 2015)[25] & UK & Cohort & 112,451 & $\begin{array}{l}\mathrm{OR}=1.04(95 \% \mathrm{CI} 1.02-1.07) \text { for lapsed; } \\
\mathrm{OR}=1.57(95 \% \mathrm{CI} 1.48-1.67) \text { for never screened }\end{array}$ \\
\hline
\end{tabular}

Figure 2. Synthesis of the literature review. (HR: hazard ratio; OR: odds ratio; PR: prevalence ratio; RR: risk ratio; Adj: adjusted).

Using the Population Registry of Reggio Emilia municipality, we identified mothers of girls living in Reggio Emilia. The Population Registry collects information on mothers' educational level, citizenship, family composition, and number and order of siblings. Household composition for girls resident in other municipalities could not be set up, as Population Registries were not available. Mothers were also classified in accordance with their screening history, by linkage to public screening programme archives data ${ }^{37}$ Supplementary file 1.

\section{Outcomes definition}

For our primary objective two outcomes were identified: having received at least one dose; and having completed the vaccination cycle with three doses within 36 months since the vaccination campaign, which started on 8 March 2008.

For our secondary objective (figure 1A), only mothers overdue for a Pap test in 2008 and 2009 were eligible. We defined eligible women as follows: mothers who had been invited before 1 January 2008 and still non-participating at that time (overdue for Pap test on 1 January 2008) and women who received an invitation letter between 2008 and 2009, i.e. who were eligible for the organised screening participation between 2008, the year of the vaccination campaign, and the following
2009. For the mothers who were invited for screening in 2008, we defined the outcome as performing a Pap test from 1 January 2008 to 31 March 2009 in the screening programme, i.e. during the year of the campaign and up to 3 months' later. For mothers invited in 2009, the outcome was having a Pap test within 3 months since the screening invitation letter. Mothers of girls being sisters in different cohorts were excluded.

\section{Exposure definition}

Birth cohort was the variable defining the intervention: active invitation (1997 cohort) and no active invitation (1996 cohort). This variable has been used as a determinant of overall coverage and mothers' participation in screening and as effect modifier of the other candidate determinants of vaccine coverage.

Girls' citizenship, mothers' age at birth, education level, marital status and number of pregnancies were considered as socio-economic candidate determinants. Girls' district of residence was considered as a proxy of local health services' and paediatricians' attitudes to vaccination. Mothers' participation in cervical cancer screening was considered a behavioural candidate determinant. According to invitations to cervical cancer screening up to 31 December 2007, women were classified as: non-participant, in case they never took part 
until 31 December 2008; irregular participant, in case they took part in at least one invitation; and regular participant, in case they took part in all invitations. Variables related to girls' characteristics were available for all the province, while variables related to mothers' characteristics were available only for the Reggio Emilia municipality.

\section{Statistical methods}

Vaccinated girls percentages (and 95\% confidence intervals) were calculated for the two cohorts.

We used stratified multivariate log-binomial regression models, in order to estimate differences in Relative Risk (RR) and 95\% confidence intervals (CIs) of vaccination in the two cohorts. Interaction was calculated according to citizenship, educationallevel, demographics (mother's age at birth, number of pregnancies, marital status) and adherence to screening. The effect modification was tested using a likelihood ratio test, by comparing the basic model with all covariates and the model with all covariates plus the term of interaction between one covariate and the cohort.

For the secondary objective, we assessed the RR of receiving a Pap test after the vaccination campaign in mothers overdue for a Pap test on 1 January 2008, by use of a log-binomial regression model adjusted by citizenship, mother's age at birth and education level. As regarded this outcome, we also reported models stratified by education level. A log-binomial regression model adjusted by citizenship, mother's age at birth and education level was also performed to assess the RR of receiving a Pap test in mothers, overdue for a Pap test on 1 January 2008, who vaccinated or did not vaccinate daughters. Only women residing in the Reggio Emilia municipality were included.

Data analysis was performed using Stata IC 13. In particular, multivariate models were assessed thanks to the Generalised Linear Model package using link logarithm and family binomial (StataCorp LP, College Station, TX, USA).

\section{Ethics, funding and data sharing}

Our research did not receive any specific grant from any public funding agency, commercial companies or not-for-profit associations. The Local Ethics Committee approved the study protocol on 20 January 2016. Record linkage procedures were performed in compliance with privacy protection rules. Anonymised dataset is available on demand (paolo.giorgirossi@ausl.re.it).

\section{RESULTS}

Impact of the active invitation campaign on HPV vaccine coverage in young-adolescent girls

In 2008, Reggio Emilia resident females included 2260 girls (born in 1996) and 2307 girls (born in 1997). Examining the 2008 to 2011 archives, we found
1046 HPV vaccinations (46.3\%, 95\% CI 44.2-48.4) for 1996-born uninvited girls and 1798 vaccinations (77.9\%, 95\% CI 76.2-79.6) for 1997-born actively invited girls. Rates of three-doses-vaccinated girls were $43.9 \%$ (1996 cohort) and $74.9 \%$ (1997 cohort).

For both cohorts, vaccination coverage recorded a significant gap among six Reggio Emilia districts (table 1A, table 2A, Supplementary file 2-table 1A and Supplementary file 2-table 2A).

We collected information related to 751 mothers in the 1996 cohort, and 769 mothers in the 1997 cohort, resident in the Reggio Emilia municipality. Of mothers in the screening target age, $99.3 \%$ (99.2\% for 1996 cohort, $99.5 \%$ for 1997 cohort) have been invited at least once to a cervical screening programme before 31 December 2007 (Supplementary file 1).

\section{Impact of active invitation campaign on HPV vaccine inequalities}

In the uninvited cohort, Italian girls had a higher rate of vaccination (one dose) than foreign ones: $50.0 \%$ (CI95\% 47.7-52.2) versus $22.8 \%$ (CI95\% $18.2-$ 27.9) (table 1), RR $0.45 \quad(\mathrm{CI} 95 \%$ 0.37-0.56). In the actively invited cohort, there was no difference in vaccination rate between the two groups (RR 0.97 CI95\% $0.92-1.03) \quad$ (table 2A) (test of interaction: $\mathrm{p}<0.00005$ ).

Multivariate analysis showed an association between levels of mothers' education and daughters' vaccination in the uninvited cohort. Coverage in daughters of women with medium education-level ( 8 to 11 and $>12$ years of education, non-graduates) was higher than coverage in daughters of graduate women or with low education level ( $\leq 7$ years education). In the actively invited cohort, no significant difference was found among the three higher education levels (table 2B),

but the difference might be due to chance (test for interaction: $\mathrm{p}=0.0948$ ).

Moreover, previous participation to cervical cancer screening programmes was associated with daughters' vaccination in both cohorts, although association was stronger in the uninvited cohort (table 2B and supplementary file 2 -table 2B), also in this case the difference between the cohorts can be due to chance (test of interaction: $\mathrm{p}=0.3981$ ).

Consideringathoroughvaccinationcycle (threedoses), the resultsweresubstantiallysimilar. Theonlydifferencewasactive invitation which, compared with no invitation, still reduced the different vaccination coverage between foreign girls and Italian ones, although association between citizenship and vaccination remained significant (supplementary file 2 -table $2 \mathrm{~A}$ and supplementary file $\quad 2 \quad$-table 2 2B).

Impact of HPV vaccination campaign on mothers' participation in cervical cancer screening

Concerning our secondary objective, we analysed the impact of the vaccination campaign 
Table 1 Distribution of girls vaccinated with at least one dose of HPV vaccine for girls, services and mothers' variables included in the study, in the two birth cohorts (not invited, 1996; actively invited, 1997). In part a, all girls resident in Reggio Emilia were included, while in part b, only girls resident in the municipality of Reggio Emilia were included.

a. Reggio Emilia province

All

Residence

\begin{tabular}{|c|c|c|c|c|c|c|}
\hline Correggio & 222 & 84 & $37.8 \%$ & 253 & 188 & $74.3 \%$ \\
\hline Montecchio & 269 & 135 & $50.2 \%$ & 274 & 227 & $82.8 \%$ \\
\hline Reggio Emilia & 999 & 520 & $52.1 \%$ & 989 & 809 & $81.8 \%$ \\
\hline Scandiano & 352 & 98 & $27.8 \%$ & 356 & 202 & $56.7 \%$ \\
\hline Castelnuovo Monti & 127 & 70 & $55.1 \%$ & 119 & 109 & $91.6 \%$ \\
\hline Guastalla & 291 & 139 & $47.8 \%$ & 316 & 263 & $83.2 \%$ \\
\hline \multicolumn{7}{|l|}{ Citizenship } \\
\hline Italian & 1953 & 977 & $50.0 \%$ & 1975 & 1535 & $77.7 \%$ \\
\hline Foreign & 307 & 70 & $22.8 \%$ & 332 & 264 & $79.5 \%$ \\
\hline $\begin{array}{l}\text { b. Reggio Emilia } \\
\text { municipality }\end{array}$ & $\mathbf{N}^{\circ}$ & $\mathbf{N}^{\circ}$ & $\%$ & $\mathbf{N}^{\circ}$ & $\mathbf{N}^{\circ}$ & $\%$ \\
\hline All & 792 & 388 & $49.0 \%$ & 803 & 651 & $81.1 \%$ \\
\hline Mother identified & 715 & 357 & $49.9 \%$ & 730 & 593 & $81.2 \%$ \\
\hline Mother not identified & 77 & 31 & $40.3 \%$ & 73 & 58 & $79.5 \%$ \\
\hline \multicolumn{7}{|l|}{ Mother's age at birth } \\
\hline$<20$ & 18 & 6 & $33.3 \%$ & 22 & 18 & $81.8 \%$ \\
\hline $20-34$ & 605 & 307 & $50.7 \%$ & 584 & 479 & $82.0 \%$ \\
\hline$>34$ & 92 & 44 & $47.8 \%$ & 124 & 96 & $77.4 \%$ \\
\hline \multicolumn{7}{|l|}{ Years of education } \\
\hline$>12$, graduate & 65 & 23 & $35.4 \%$ & 78 & 66 & $84.6 \%$ \\
\hline$>12$, non-graduate & 261 & 147 & $56.3 \%$ & 280 & 225 & $80.4 \%$ \\
\hline $8-11$ & 321 & 165 & $51.4 \%$ & 318 & 265 & $83.3 \%$ \\
\hline$\leq 7$ & 65 & 22 & $33.9 \%$ & 52 & 35 & $67.3 \%$ \\
\hline Missing & 3 & 0 & $0.0 \%$ & 2 & 2 & $100.0 \%$ \\
\hline \multicolumn{7}{|l|}{ Citizenship } \\
\hline Italian & 609 & 326 & $53.5 \%$ & 608 & 493 & $81.1 \%$ \\
\hline Foreign & 106 & 31 & $29.2 \%$ & 122 & 100 & $82.0 \%$ \\
\hline \multicolumn{7}{|l|}{ Single parent } \\
\hline Yes & 87 & 44 & $50.6 \%$ & 88 & 74 & $84.1 \%$ \\
\hline No & 628 & 313 & $49.8 \%$ & 642 & 519 & $80.8 \%$ \\
\hline \multicolumn{7}{|l|}{ Sibling } \\
\hline No & 161 & 85 & $52.8 \%$ & 162 & 130 & $80.2 \%$ \\
\hline Yes & 554 & 272 & $49.1 \%$ & 568 & 463 & $81.5 \%$ \\
\hline \multicolumn{7}{|l|}{ Screening history } \\
\hline Non-participant & 89 & 29 & $32.6 \%$ & 90 & 62 & $68.9 \%$ \\
\hline Irregular participant & 335 & 164 & $49.0 \%$ & 341 & 283 & $83.0 \%$ \\
\hline Regular participant & 285 & 162 & $56.8 \%$ & 295 & 245 & $83.1 \%$ \\
\hline Missing & 6 & 2 & $33.3 \%$ & 4 & 3 & $75.0 \%$ \\
\hline
\end{tabular}

\begin{tabular}{lll}
\hline All & \multicolumn{2}{l}{ Vaccinated (at least one dose) } \\
\hline $\mathbf{N}^{\circ}$ & $\mathbf{N}^{\circ}$ & $\%$
\end{tabular}

2260

1046

$46.3 \%$

All

$\mathbf{N}^{\circ}$

2307

$\mathrm{N}^{\circ}$

Vaccinated (at least one dose)

1798

$77.9 \%$

\begin{tabular}{l}
\hline All \\
\hline $\mathbf{N}^{\circ}$ \\
2260
\end{tabular}

R

Citizenship

\section{b. Reggio Emilia} municipality

Birth cohort 1997

\section{Birth cohort 1996}

\section{All}


Table 2 Relative risk (RR) of being vaccinated with at least one dose for girls in the two birth cohorts (not invited, 1996; actively invited, 1997). Multivariate log-binomial regression models stratified by birth cohort and adjusted by citizenship, educational level, demographics (mother's age at birth, number of pregnancies, marital status) and adherence to screening were performed. In part a, all girls resident in Reggio Emilia were included while in part b, only girls resident in the municipality of Reggio Emilia were included.

\begin{tabular}{|c|c|}
\hline Birth cohort $1996(n=2260)$ & Birth cohort $1997(n=2307)$ \\
\hline $\begin{array}{l}\text { Vaccinated (at least one } \\
\text { dose) }\end{array}$ & Vaccinated (at least one dose) \\
\hline $95 \% \mathrm{Cl}$ & $95 \% \mathrm{Cl}$ \\
\hline
\end{tabular}

\section{Residence (test for interaction $\mathrm{p}=0.1005$ )}

$\begin{array}{lllll}\text { Correggio } & 1 & - & 1 & - \\ \text { Montecchio } & 1.26 & (1.03 \text { to } 1.55) & 1.12 & \text { (1.02 to } 1.22) \\ \text { Reggio Emilia } & 1.35 & (1.14 \text { to } 1.61) & 1.10 & \text { (1.02 to } 1.19) \\ \text { Scandiano } & 0.71 & (0.56 \text { to } 0.90) & 0.76 & \text { (0.68 to } 0.86) \\ \text { Castelnuovo Monti } & 1.42 & (1.13 \text { to } 1.78) & 1.24 & \text { (1.13 to } 1.35) \\ \text { Guastalla } & 1.25 & (1.03 \text { to } 1.54) & 1.12 & \text { (1.03 to } 1.23)\end{array}$

Citizenship (test for interaction $p<0.00005$ )

\begin{tabular}{|c|c|c|c|c|}
\hline Italian & 1 & - & 1 & - \\
\hline Foreign & 0.45 & (0.37 to 0.56$)$ & 0.97 & (0.92 to 1.03$)$ \\
\hline b. Reggio Emilia municipality & $(n=706)$ & & $(n=724)$ & \\
\hline \multicolumn{5}{|l|}{$\begin{array}{l}\text { Mother's age at birth } \\
\text { (test for interaction } \mathrm{p}=0.5382 \text { ) }\end{array}$} \\
\hline$<20$ & 1 & - & 1 & - \\
\hline $20-34$ & 1.26 & (0.67 to 2.38$)$ & 0.98 & (0.78 to 1.22$)$ \\
\hline$>34$ & 1.28 & (0.66 to 2.48$)$ & 0.93 & (0.73 to 1.18$)$ \\
\hline \multicolumn{5}{|l|}{$\begin{array}{l}\text { Years of education } \\
\text { (test for interaction } \mathrm{p}=0.0948 \text { ) }\end{array}$} \\
\hline$>12$, graduate & 1 & - & 1 & - \\
\hline$>12$, non-graduate & 1.55 & (1.10 to 2.19$)$ & 0.95 & (0.85 to 1.06$)$ \\
\hline $8-11$ & 1.61 & (1.14 to 2.28$)$ & 0.98 & (0.88 to 1.10$)$ \\
\hline$\leq 7$ & 1.06 & $(0.66-1-69)$ & 0.82 & (0.66 to 1.01$)$ \\
\hline \multicolumn{5}{|c|}{ Single parent (test for interaction $\mathrm{p}=0.4809$ ) } \\
\hline No & 1 & - & 1 & - \\
\hline Yes & 1.06 & (0.86 to 1.31$)$ & 1.08 & (0.98 to 1.19$)$ \\
\hline \multicolumn{5}{|c|}{ Sibling (test for interaction $\mathrm{p}=0.5543$ ) } \\
\hline No & 1 & - & 1 & - \\
\hline Yes & 0.99 & (0.83 to 1.16$)$ & 1.04 & (0.95 to 1.14$)$ \\
\hline \multicolumn{5}{|c|}{ Screening history (test for interaction $p=0.3981$ ) } \\
\hline Non-participant & 1 & - & 1 & - \\
\hline Irregular participant & 1.49 & (1.09 to 2.04$)$ & 1.19 & (1.03 to 1.38$)$ \\
\hline Regular participant & 1.72 & (1.26 to 2.36 ) & 1.20 & (1.04 to 1.40$)$ \\
\hline
\end{tabular}

exposure on participation in organised screening by mothers overdue for a Pap test on 1 January 2008.

No overall differences in participation were recorded in the two cohorts. Mothers who had their daughters vaccinated at least with one dose, recorded higher participation if compared with mothers who didn't have their daughters vaccinated

$(73.1 \%$ and $61.5 \%$ respectively, RR $1.18 \quad 95 \%$ CI
1.07-1.30) (table 3). Similar results were found in a thorough immunisation cycle (supplementary file 2 -table 3 ). Furthermore, by stratifying mothers according to their educational level, we observed that graduate mothers of actively invited girls showed a stronger likelihood of participating in screening programmes after the vaccination campaign period, if compared 
Table 3 Relative risk (RR) of receiving a Pap test after the vaccination campaign in mothers overdue for a Pap test on 1 January 2008. Two log-binomial regression models adjusted by mother's age at birth, education level and citizenship was performed: the first to test the effect of the active vaccination campaign (1997 cohort) versus no invitation (1996 cohort) and the second to test the association between daughter's vaccination. Second, the RR of receiving a Pap test after the vaccination campaign among mother's education strata was assessed performing a stratified log-binomial regression model adjusted by mother's age at birth and citizenship. Only women resident in the Reggio Emilia municipality were included. [Low $\leq 11$ years of education; Medium $>12$, non-graduate; High $>12$, graduate]

\begin{tabular}{|c|c|c|c|}
\hline \multirow[b]{2}{*}{ Mothers overdue for a Pap test on 1 January 2008} & \multicolumn{3}{|c|}{ Pap test participation } \\
\hline & $(\%)$ & RR & $95 \% \mathrm{Cl}$ \\
\hline \multicolumn{4}{|l|}{ Mother's age at birth } \\
\hline$<20(\mathrm{n}=4)$ & 50.0 & 1 & - \\
\hline $20-34(n=736)$ & 70.7 & 1.26 & (0.47 to 3.36$)$ \\
\hline$>34(n=213)$ & 64.8 & 0.75 & (0.42 to 3.02$)$ \\
\hline \multicolumn{4}{|l|}{ Years of education } \\
\hline$>12$, graduate $(n=101)$ & 81.2 & 1 & - \\
\hline$>12$, non-graduate $(n=345)$ & 75.4 & 0.91 & (0.81 to 1.02$)$ \\
\hline$\leq 12(n=505)$ & 62.6 & 0.76 & (0.67 to 0.85$)$ \\
\hline \multicolumn{4}{|l|}{ Citizenship } \\
\hline Italian $(n=815)$ & 69.7 & 1 & - \\
\hline Foreign $(n=138)$ & 66.7 & 0.99 & (0.87 to 1.13$)$ \\
\hline \multicolumn{4}{|l|}{ Cohort } \\
\hline $1996(n=472)$ & 68.0 & 1 & - \\
\hline $1997(n=481)$ & 70.5 & 1.04 & (0.96 to 1.13$)$ \\
\hline \multicolumn{4}{|l|}{ Daughter's vaccination status (at least one dose) } \\
\hline Mothers who did not vaccinate $(n=317)$ & 61.5 & 1 & - \\
\hline $\begin{array}{l}\text { Mothers who vaccinated } \\
(n=636)\end{array}$ & 73.1 & 1.18 & (1.07 to 1.30$)$ \\
\hline
\end{tabular}

Stratified analyses

by mother's educational level (test for interaction $\mathrm{p}=0.0402$ )

Low $(n=505)$

$\begin{array}{llll}1996 & 62.1 & 1 & - \\ 1997 & 63.1 & 1.01 & (0.88 \text { to } 1.16) \\ \begin{array}{l}\text { Medium }(n=345) \\ 1996\end{array} & 75.9 & & - \\ 1997 & 74.3 & 1 & (0.86 \text { to } 1.11) \\ \text { High }(n=95) & & & - \\ 1996 & 69.8 & 1 & (1.04 \text { to } 1.56) \\ 1997 & 89.7 & 1.27 & \end{array}$

with those of the uninvited cohort (table 3).

invited cohort recorded higher immunisation uptake and reduced socio-economic inequalities, compared

No difference was found in screening participation between the two cohorts, when stratifying mothers according to daughter's vaccination (test of interaction: $p=0.730$ for vaccination with at least one dose, $p=0.581$ with three doses).

\section{DISCUSSION}

Two birth cohorts were simultaneously exposed to two different vaccination strategies. The actively with the not actively invited cohort. Active invitation considerably decreased inequalities due to immigration status and educational level and it less markedly reduced association with mother's participation in screening, although it had almost no impact geographically (ie, increase was almost the same for all districts). Moreover, the active invitation for daughters' vaccination acted as a reminder for mothers overdue for cervical cancer screening, but only among women graduates. 
Our work is one of the few Italian studies which compares immunisation coverage between vaccination strategies with and without active invitation. Our results are consistent with those reached by Giambi $e t a l \mathrm{~s}^{30}$ study and a previous systematic review, ${ }^{31}$ although considering secondary prevention programmes.

The association between HPV vaccination and cervical cancer screening participation gets high relevance in a public health perspective for two main reasons. First, it sharply reduces the effectiveness of intervention on the burden of disease. As a matter of fact, the vast majority of invasive cervical cancers occurring in industrialised countries are now affecting under-screened women. Therefore, failing to vaccinate them would almost reduce the impact of vaccination in a public health perspective. Second, vaccination could play either a positive or negative role in screening participation for mothers of vaccine target cohorts and young adult women who are themselves the target of opportunistic vaccination.

In order to further analyse such connections, several study designs have been adopted, as shown by figure 2 . Some authors stressed the association between vaccination in young adult women and screening habits. We defined this as the 'mothers on mothers' effect. Unfortunately, such studies, cannot yet be performed on cohorts that have been targeted by mass vaccination campaigns. ${ }^{9} 1013-16$ Other authors described the association between mothers' screening habits and daughters' vaccination, suggesting that a preventive attitude by mothers would determine their daughters' attitude, once they have reached screening target age. We defined it as the 'mothers on daughters' effect. ${ }^{17-24}$ Finally, some other studies observed the impact of the vaccination campaign on mothers' screening habits. We defined it as the 'daughters on mothers' effect. ${ }^{25}$

\section{Vaccination and socio-demographic characteristics}

In the uninvited cohort of Reggio Emilia province resident girls, a lower immunisation coverage among foreigners was recorded in comparison to Italians. Moreover, mothers at the lowest and highest education level were less likely to have their daughters vaccinated, in comparison to mothers at medium education level. Despite vaccine being free, our results were consistent with several other studies reporting girls from ethnic minorities and more deprived areas showing lower complete immunisation series in comparison to more advantaged girls. ${ }^{12}{ }^{38-46}$ On the contrary, in the actively invited cohort we found no difference in one-dose vaccination, either considering citizenship or education level. Taking into account the thorough immunisation cycle, foreign mothers still vaccinate to a lesser extent, when compared with Italian mothers, as less educated mothers do in comparison to graduate mothers, but with a significantly reduced gap. A Canadian population-based study showed that school-based vaccination programmes can lead to high vaccination coverage even in areas populated by a higher proportion of ethnic minorities. ${ }^{47}$

\section{Vaccination and mother's participation in screening}

A recent review reported an association between high HPV vaccination and ethnic majority populations, high socio-economic status, as well as mother's preventive attitude assessed through cervical screening participation and previous vaccination during childhood. ${ }^{46}$ The relationship between HPV vaccination and cervical cancer screening participation was thoroughly discussed. Recently, many studies reported a positive association between HPV vaccination history and attendance of cervical cancer screening (figure 2). ${ }^{91013-1639}$

We could not assess screening uptake in vaccinated and non-vaccinated girls, because cohorts targeted by the vaccination campaign were still too young for screening. Therefore, just like many previous studies $\operatorname{did},{ }^{17-24}$ we considered an association between mothers' participation history in cervical screening programmes as a proxy of future attitudes to screening of girls (figure 1B). Our findings were consistent with recent studies, ${ }^{17-24}$ showing that immunisation coverage in daughters of mothers regularly and irregularly participating in cervical cancer screening was higher than immunisation coverage in daughters of non-participating women.

Evidence of an impact of parental attitudes towards preventive measures in daughters' decision-making was widely reported. ${ }^{23} 244849$ A qualitative systematic review described girls as mainly 'HPV vaccine passive recipients' and stressed the role of parents affected by concerns about the safety/efficacy of vaccines and sexual mores. ${ }^{50}$ Our study showed an active invitation strategy be less effective in reducing differences related to mother's preventive behaviours, than socio-demographic inequalities. Nevertheless, increasing overall vaccination coverage directly reduced the absolute values of non-vaccinated girls in both groups.

\section{Active invitation campaign - 'Daughter on mother' effect}

Concerning our secondary objective we analysed whether exposure to the active invitation campaign could increase mother's participation in cervical cancer screening (figure 1A). We recorded no difference in Pap test participation after the campaign period between overall previous non-participant mothers related to the two birth cohorts. In addition, although daughters' vaccinations were associated with higher screening participation, difference turned out almost the same in the two cohorts. Nevertheless, stratifying by educational level we could observe an effect of vaccination active invitation on mother's screening participation only among graduate mothers overdue for a Pap test. A new British study recorded an increased screening participation among mothers exposed to an active national vaccination programme. ${ }^{25}$ The different impact of receiving an invitation letter for daughter's vaccination in graduated mothers and non-graduated ones might be connected to how the link between HPV vaccination and cervical cancer screening was illustrated by both invitation letter and information leaflet. In particular, the Reggio Emilia 
LHA invitation letter gave brief information about HPV vaccine preventing cervical cancer, while a short indication of the necessity of screening by vaccinated women was only reported in a separate leaflet. The correlation between vaccine and screening probably required a higher level of health literacy. An invitation letter, which more clearly explained primary and secondary cervical cancer prevention programmes and written in different languages, should be considered to further test its possible impact on the screening awareness and participation, also by disadvantaged population groups.

\section{Strengths and limitations}

Availability of data exclusively related to LHA-organised screening represents the major limitation of our study.

Although $75 \%$ total screening coverage was represented, which is a much higher level than the average Italian one, a significant part of Pap tests was not included. ${ }^{27}$

Moreover, only cohorts residing in one province and because of screening association/effect residing in one municipality were included, thus limiting both eligibility of subjects and generalisability of results.

The quasi-experimental comparison of two vaccination strategies in a cohort study design and the use of routinely collected data are to be mentioned as our strength. They allowed us to assess the real participation of mothers and daughters in primary and secondary prevention programmes, which is a more reliable value than a self-reported willingness to participate.

\section{CONCLUSIONS}

According to our study, active invitation was effective in increasing overall HPV immunisation coverage and reducing both socio-demographic inequalities and association with mother's preventive behaviours. The impact of the vaccination campaign as a reminder for mothers' screening was only detectable in highly educated women.

\section{Author affiliations}

${ }^{1}$ Interinstitutional Epidemiology Unit, Local Health Authority of Reggio Emilia, Reggio Emilia, Italy

${ }^{2}$ Arcispedale Santa Maria Nuova-IRCCS, Reggio Emilia, Italy

${ }^{3}$ Department of Biomedical, Metabolic and Neural Sciences, University of Modena and Reggio Emilia, Modena, Italy

${ }^{4}$ Romagna Cancer Registry, Istituto Scientifico Romagnolo per lo Studio e la Cura dei Tumori (IRST)-IRCCS, Meldola, Italy

${ }^{5}$ Coordination Screening Centre, Local Health Authority of Reggio Emilia, Reggio Emilia, Italy

${ }^{6}$ Public Health Service, Local Health Authority of Reggio Emilia, Reggio Emilia, Italy ${ }^{7}$ Directorate General for Health and Social Policy - Emilia-Romagna Region, Bologna, Italy

${ }^{8}$ Community paediatrics, Local Health Authority of Reggio Emilia, Reggio Emilia, Italy

Acknowledgements Authors would like to thank Dr Daniela Masi (Local Health Authority of Reggio Emilia) for linguistic support.

Contributors ACF, MGP, FB, FV and PGR contributed to the study concepts and study design. PGR, FB and FV retrieved initial references for the background. FB, $\mathrm{CC}, \mathrm{CP}$ and LM worked on quality control of data and linkage between datasets and FB analysed data. All the authors participated in the interpretation of the results. FB, FV and PGR drafted the manuscript and PGR and ACF revised important contents in the article. FV worked on manuscript editing. All the authors have seen and approved the final version of the manuscript. PGR guarantees for data integrity.

Competing interests All authors filled out ICMJE uniform disclosure form at www. icmje.org/coi_disclosure.pdf and declared: no support from any organization for thesubmitted manuscript; no financial relationships with any organizations that might have an interest in the submitted work in the previous three years; no other relationships or activities that could appear to have influenced the submitted work.

Ethics approval Ethics Committee of the Local Health Authoritiy of Reggio Emilia.

Provenance and peer review Not commissioned; externally peer reviewed.

Data sharing statement Anonymised dataset is available by emailing paolo. giorgirossi@ausl.re.it.

Open Access This is an Open Access article distributed in accordance with the Creative Commons Attribution Non Commercial (CC BY-NC 4.0) license, which permits others to distribute, remix, adapt, build upon this work non-commercially, and license their derivative works on different terms, provided the original work is properly cited and the use is non-commercial. See: http://creativecommons.org/ licenses/by-nc/4.0/

(c) Article author(s) (or their employer(s) unless otherwise stated in the text of the article) 2017. All rights reserved. No commercial use is permitted unless otherwise expressly granted.

\section{REFERENCES}

1. IARC Working Group on the Evaluation of Carcinogenic Risks to Humans. IARC Monogr Eval Carcinog risks to humans. Human papillomaviruses. 2007;90:1-636.

2. WHO. Comprehensive cervical cancer control. A guide to essential practice. Geneva, 2014 http://apps.who.int/iris/bitstream/10665/ 144785/1/9789241548953_eng.pdf (accessed 2 Nov 2016).

3. European Centre for Disease Prevention and Control. Introduction of HPV vaccines in European Union countries: an update. Stockholm, 2012 http://ecdc.europa.eu/en/publications/Publications/20120905_ GUI_HPV_vaccine_update.pdf (accessed 2 Nov 2016).

4. Arbyn M, Anttila A, Jordan J, et al. European Guidelines for Quality Assurance in Cervical Cancer Screening. Second edition-summary document. Annals of Oncology 2010;21:448-58.

5. Bruni L, Barrionuevo-Rosas L, Albero G, et al. Human Papillomavirus and related diseases in Europe. 2015. Summary Report 2015-04-08 http://www.hpvcentre.net/statistics/reports/XEX.pdf (accessed 2 Nov 2016).

6. Giorgi Rossi P, Carozzi F, Federici A, et al. Italian Screening in HPV vaccinated girls Consensus Conference group. Cervical cancer screening in women vaccinated against human papillomavirus infection: Recommendations from a consensus conference. Prev Med 2017;98:21-30.

7. Brotherton JML, Mullins RM. Will vaccinated women attend cervical screening? A population based survey of human papillomavirus vaccination and cervical screening among young women in Victoria, Australia. Cancer Epidemiol 2012;36:298-302.

8. Anhang Price R, Koshiol J, Kobrin S, et al. Knowledge and intention to participate in cervical cancer screening after the human papillomavirus vaccine. Vaccine 2011;29:4238-43.

9. Herweijer E, Feldman AL, Ploner A, et al. The participation of HPV-vaccinated women in a national cervical screening program: population-based cohort study. PLoS One 2015;10:e0134185.

10. Sauer AG, Jemal A, Simard EP, et al. Differential uptake of recent Papanicolaou testing by HPV vaccination status among young women in the United States, 2008-2013. Cancer Epidemiol 2015;39:650-5.

11. Giorgi Rossi P, Baldacchini F, Ronco G. The possible effects on socio-economic Inequalities of Introducing HPV testing as primary test in cervical cancer screening programs. Front Oncol 2014;4:20.

12. Beer H, Hibbitts S, Brophy S, et al. Does the HPV vaccination programme have implications for cervical screening programmes in the UK? Vaccine 2014;32:1828-33.

13. Palmer TJ, McFadden M, Pollock KGJ, et al. HPV immunisation and increased uptake of cervical screening in Scottish women; observational study of routinely collected national data. Br J Cancer 2016;114:576-81.

14. Boone SD, Pinkston CM, Baumgartner KB, et al. Associations between prior HPV4 vaccine doses and cervical cancer screening participation. Cancer Epidemiol 2016;42:108-14. 
15. Paynter CA, Van Treeck BJ, Verdenius I, et al. Adherence to cervical cancer screening varies by human papillomavirus vaccination status in a high-risk population. Prev Med Rep 2015;2:711-6.

16. Budd AC, Brotherton JML, Gertig DM, et al. Cervical screening rates for women vaccinated against human papillomavirus. Med J Aust 2014;201:279-82.

17. Hansen BT, Campbell S, Burger E, et al. Correlates of HPV vaccine uptake in school-based routine vaccination of preadolescent girls in Norway: a register-based study of 90,000 girls and their parents. Prev Med 2015;77:4-10.

18. Markovitz AR, Song JY, Paustian ML, et al. Association between maternal preventive care utilization and adolescent vaccination: it's not just about Pap testing. J Pediatr Adolesc Gynecol 2014;27:29-36.

19. Steens A, Wielders $\mathrm{CCH}$, Bogaards JA, et al. Association between human papillomavirus vaccine uptake and cervical cancer screening in the Netherlands: implications for future impact on prevention. Int $J$ Cancer 2013;132:932-43.

20. Spencer Nee Pilkington AM, Brabin L, Verma A, et al. Mothers' screening histories influence daughters' vaccination uptake: an analysis of linked cervical screening and human papillomavirus vaccination records in the North West of England. Eur $J$ Cancer 2013;49:1264-72.

21. Hechter RC, Chao C, Sy LS, Ls S, et al. Quadrivalent human Papillomavirus vaccine uptake in adolescent boys and maternal utilization of preventive care and history of sexually transmitted infections. Am J Public Health 2013;103:e63-8.

22. Lutringer-Magnin $\mathrm{D}$, Cropet $\mathrm{C}$, Barone $\mathrm{G}$, et al. HPV vaccination among French girls and women aged $14-23$ years and the relationship with their mothers' uptake of Pap smear screening: a study in general practice. Vaccine 2013;31:5243-9.

23. Lefevere $\mathrm{E}$, Hens $\mathrm{N}$, Theeten $\mathrm{H}$, et al. Like mother, like daughter? Mother's history of cervical cancer screening and daughter's human Papillomavirus vaccine uptake in Flanders (Belgium). Vaccine 2011;29:8390-6.

24. Chao C, Slezak JM, Coleman KJ, et al. Papanicolaou screening behavior in mothers and human Papillomavirus vaccine uptake in adolescent girls. Am J Public Health 2009;99:1137-42.

25. Spencer AM, Roberts SA, Verma A, et al. Effect of human Papillomavirus vaccination of daughters on the cervical screening uptake of their non-vaccinated mothers. Eur J Public Health 2015;25:1097-100.

26. Marlow LAV, Waller J, Wardle J. Sociodemographic predictors of HPV testing and vaccination acceptability: results from a population-representative sample of British women. J Med Screen 2008;15:91-6.

27. Carrozzi G, Sampaolo L, Bolognesi L, et al. Cancer screening uptake: association with individual characteristics, geographic distribution, and time trends in Italy. Epidemiol Prev 2015;39:9-18.

28. Owsianka B, Gańczak M. Evaluation of human papilloma virus (HPV) vaccination strategies and vaccination coverage in adolescent girls worldwide. Przegl Epidemiol 2015;69:53151-85

29. von Karsa L, Arbyn A, De Vuyst $\mathrm{H}$, et al. European guidelines for quality assurance in cervical cancer screening. 2nd ed. Supplements. Luxembourg, $2015 \mathrm{http}: / /$ www.gisci.it/documenti/news/ EW0115451ENN_002.pdf (accessed 2 Nov 2016).

30. Giambi C, Del Manso M, D'Ancona F, et al. Actions improving HPV vaccination uptake - Results from a national survey in Italy. Vaccine 2015;33:2425-31.

31. Ferroni E, Camilloni L, Jimenez B, et al. How to increase uptake in oncologic screening: a systematic review of studies comparing population-based screening programs and spontaneous access. Prev Med 2012;55:587-96.

32. Palencia L, Espelt A, Rodriguez-Sanz M, et al. Socio-economic inequalities in breast and cervical cancer screening practices in Europe: influence of the type of screening program. Int J Epidemiol 2010;39:757-65
33. Paul-Ebhohimhen $\mathrm{V}$, Huc $\mathrm{S}$, Tissington $\mathrm{H}$, et al. HPV vaccination: vaccine acceptance, side effects and screening intentions. Community Pract 2010;83:30-3

34. Goldhaber-Fiebert JD, Stout NK, Salomon JA, et al. Costeffectiveness of cervical cancer screening with human Papillomavirus DNA testing and HPV-16,18 vaccination. J Natl Cancer Inst 2008:100:308-20.

35. La conferenza permanente per i rapporti tra lo stato le regioni e le provincie autonome di Trento e Bolzano. Strategia per l'offerta attiva del vaccino contro l'infezione da HPV in Italia. 2007. http://www. statoregioni.it/Documenti/DOC_016696_264 csr.pdf (accessed 2 Nov 2016).

36. Bertozzi N, Carrozzi G. I dati di sorveglianza PASSI: confronto fra Emilia-Romagna e Italia. http://salute.regione.emilia-romagna.it/ screening/tumori-femminili/documentazione/atti-relativi-a-convegnie-seminari-regionali/la-sorveglianza-epidemiologica-dello-screeningdei-tumori-del-collo-dell2019utero-nella-regione-emilia-romagna/ Bertozzi.pdf (accessed 2 Nov 2016).

37. Giorgi Rossi P, Caroli S, Mancini S, et al. FFE-R cervical cancer screening and pathology registry group. Screening history of cervical cancers in Emilia-Romagna, Italy: defining priorities to improve cervical cancer screening. Eur J Cancer Prev 2015;24:128-34.

38. Hughes A, Mesher D, White J, et al. Coverage of the English national human papillomavirus (HPV) immunisation programme among 12 to 17 year-old females by area-level deprivation score, England, 2008 to 2011. Eurosurveillance 2014;19:20677.

39. Bowyer HL, Dodd RH, Marlow LAV, et al. Association between human papillomavirus vaccine status and other cervical cancer risk factors. Vaccine 2014:32:4310-6.

40. Smith LM, Brassard P, Kwong JC, et al. Factors associated with initiation and completion of the quadrivalent human papillomavirus vaccine series in an Ontario cohort of grade 8 girls. BMC Public Health 2011;11:645.

41. Sacks RJ, Copas AJ, Wilkinson DM, et al. Uptake of the HPV vaccination programme in England: a cross-sectional survey of young women attending sexual health services. Sex Transm Infect 2014:90:315-21.

42. Tanton C, Soldan K, Beddows S, et al. High-risk human Papillomavirus (HPV) infection and cervical cancer prevention in Britain: evidence of differential uptake of interventions from a probability survey. Cancer Epidemiol Biomarkers Prev 2015;24:842-53.

43. Fisher H, Audrey S, Mytton JA, et al. Examining inequalities in the uptake of the school-based HPV vaccination programme in England: a retrospective cohort study. J Public Health 2014;36:36-45.

44. Spencer AM, Roberts SA, Brabin L, et al. Sociodemographic factors predicting mother's cervical screening and daughter's HPV vaccination uptake. J Epidemiol Community Health 2014;68:571-7.

45. Sinka K, Kavanagh K, Gordon R, et al. Achieving high and equitable coverage of adolescent HPV vaccine in Scotland. J Epidemiol Community Health 2014;68:57-63.

46. Casadevante Fde V, Gil Cuesta J, Cantarero-Arévalo L. Determinants in the uptake of the human papillomavirus vaccine: a systematic review based on European studies. Front Oncol 2015;5:141.

47. Drolet M, Deeks SL, Kliewer E, et al. Can high overall human papillomavirus vaccination coverage hide sociodemographic inequalities? An ecological analysis in Canada. Vaccine 2016:34:1874-80.

48. Ogilvie G, Anderson M, Marra F, et al. A population-based evaluation of a publicly funded, school-based HPV vaccine program in British Columbia, Canada: parental factors associated with HPV vaccine receipt. PLoS Med 2010;7:e1000270.

49. Monnat SM, Wallington SF. Is there an association between maternal Pap test use and adolescent human Papillomavirus vaccination? $J$ Adolesc Health 2013;52:212-8.

50. Ferrer HB, Trotter C, Hickman M, et al. Barriers and facilitators to HPV vaccination of young women in high-income countries: a qualitative systematic review and evidence synthesis. BMC Public Health 2014:14:700. 